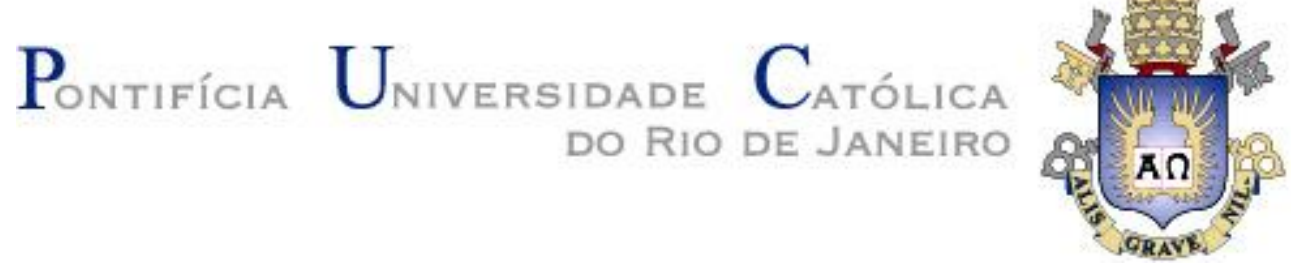

Márcio Nascimento de Souza Leão

\title{
Modelagem em Experimentos com Mistura e Mistura-Processo
}

Dissertação apresentada como requisito parcial para obtenção do grau de Mestre pelo Programa de PósGraduação em Engenharia de Produção do Departamento de Engenharia Industrial da PUC-Rio.

Orientador: Prof. Antonio Fernando de Castro Vieira 
Márcio Nascimento de Souza Leão

\author{
Modelagem em Experimentos com \\ Mistura e Mistura-Processo
}

\begin{abstract}
Dissertação apresentada como requisito parcial para obtenção do grau de Mestre pelo Programa de PósGraduação em Engenharia de Produção do Departamento de Engenharia Industrial da PUC-Rio. Aprovada pela Comissão Examinadora abaixo assinada.
\end{abstract}

Prof. Antonio Fernando de Castro Vieira Orientador Departamento de Engenharia Industrial - PUC-Rio

Prof. Luiz Henrique Abreu Dal Bello Co-orientador Departamento de Engenharia Industrial - PUC-Rio

Prof. Eugenio Kahn Epprecht Departamento de Engenharia Industrial - PUC-Rio

Prof. Flávia Cesar Teixeira Mendes Departamento de Engenharia Industrial - PUC-Rio

Prof. José Eugenio Leal Coordenador Setorial do Centro Técnico e Científico - PUC-Rio 
Todos os direitos reservados. É proibida a reprodução total ou parcial do trabalho sem autorização da universidade, do autor e do orientador.

Márcio Nascimento de Souza Leão

Graduou-se em Engenharia Mecânica e de Automóveis pelo Instituto Militar de Engenharia (IME) no ano de 2005.

Ficha Catalográfica

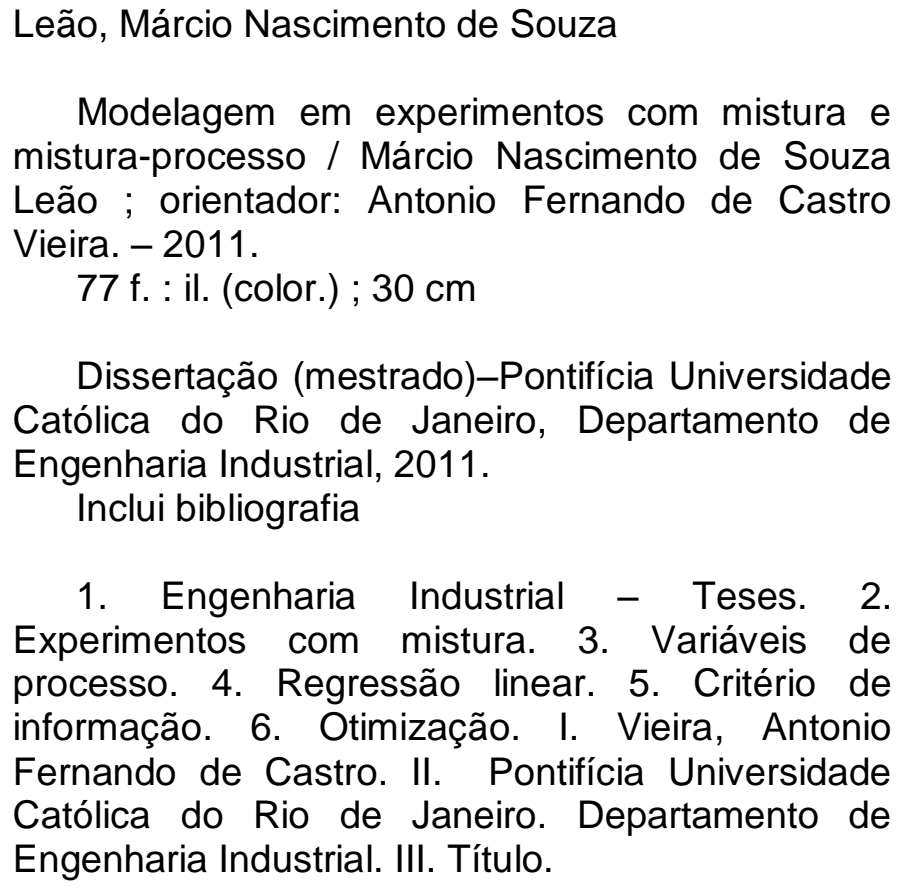

CDD: 658.5 
A minha amada esposa, pelo incentivo e apoio nos bons e difíceis momentos de nossas vidas. 


\section{Agradecimentos}

A Deus, pela oportunidade concedida.

Ao Exército Brasileiro, por permitir a realização deste curso.

Ao meu orientador Professor Antonio Fernando de Castro Vieira, pela orientação ímpar na realização deste trabalho.

Ao meu co-orientador Luiz Henrique Abreu Dal Bello, por todo o apoio.

Ao meu amigo Gustavo Simão Rodrigues, pelo incentivo em realizar este curso.

Aos professores que participaram da Comissão Examinadora.

A todos os professores e funcionários do Departamento de Engenharia Industrial, pelos ensinamentos e pela ajuda.

A minha esposa Karine, pela compreensão e apoio moral.

Por fim, os meus pais, pelo apoio e suporte necessário. 


\section{Resumo}

Leão, Márcio Nascimento de Souza; Vieira, Antonio Fernando de Castro. Modelagem em Experimentos com Mistura e Mistura-Processo. Rio de Janeiro, 2011. 77p. Dissertação de Mestrado - Departamento de Engenharia Industrial, Pontifícia Universidade Católica do Rio de Janeiro.

Nesta dissertação é apresentada uma síntese das técnicas estatísticas necessárias ao planejamento e análise de experimentos com mistura e misturaprocesso, e é apresentada uma metodologia original indicada para a seleção de modelos, especialmente para aqueles com forte colinearidade entre os níveis dos componentes da mistura, utilizando um critério baseado na Teoria da Informação. A metodologia é ilustrada com dois exemplos da literatura. Aplicando esta metodologia aos dois estudos de caso, tem-se como objetivo a seleção de modelos melhores dos que os apresentados inicialmente nos dois exemplos. Com os modelos definidos, foram determinadas as proporções ótimas dos componentes de mistura e os níveis ótimos das variáveis de processo para cada um dos exemplos. A metodologia desenvolvida para seleção de modelos, consistindo de duas etapas, provou ser eficiente nos dois casos estudados.

\section{Palavras-chave}

Experimentos com mistura; variável de processo; regressão linear; critério de informação; otimização. 


\section{Abstract}

Leão, Márcio Nascimento de Souza; Vieira, Antonio Fernando de Castro (Advisor). Modeling in Mixture and Mixture-Process Experiments. Rio de Janeiro, 2011. 77p. MSc. Dissertation - Departamento de Engenharia Industrial, Pontifícia Universidade Católica do Rio de Janeiro.

This dissertation presents a summary of the statistical techniques necessary for planning and analysis of mixture and mixture-process experiments and presented an original methodology is indicated for the selection of models, especially for those with high collinearity between the components levels of the mixture, using a criterion based on information theory. The methodology is illustrated with two examples from the literature. Applying this methodology to two case studies has as its objective the selection of the best models than presented in the originally two examples. With the models defined, were determined optimal proportions of the mix components and the optimal levels of the process variables for each two examples. The methodology developed for model selection, which consists of two steps, proved to be efficient in both studied cases.

\section{Keywords}

Mixture experiments; process variable; linear regression; information criterion; optimization. 


\section{Sumário}

1 Introdução 15

1.1. Justificativa do Trabalho 16

$\begin{array}{ll}\text { 1.2. Objetivos do Trabalho } & 17\end{array}$

$\begin{array}{ll}\text { 1.2.1. Objetivo Principal } & 17\end{array}$

$\begin{array}{ll}\text { 1.2.2. Objetivos Secundários } & 17\end{array}$

1.3. Estrutura do Trabalho 18

1.4. Softwares Utilizados 19

2 Experimentos com Mistura $\quad 21$

2.1. Modelos de Scheffé para Experimentos com Mistura 22

2.2. Pseudocomponentes 23

2.3. Exemplo $1 \quad 25$

3 Experimentos Mistura-Processo 28

3.1. Modelo para Variáveis de Processo 29

3.2. Modelos de Experimentos com Mistura incluindo Variáveis de Processo 29

3.3. Exemplo 2

4 Critérios de Seleção e Metodologia Proposta 35

4.1. Critérios de Informação 35

4.1.1. Critério de Akaike $(A / C) \quad 36$

4.1.2. Critério $A / C_{C} \quad 37$

4.1.3. Conceito de Indiferença de $A / C_{C} \quad 37$

4.2. PRESS e MSE 38

4.3. Metodologia Proposta 39

4.4. Estudo de Caso 1

4.5. Estudo de Caso $2 \quad 49$

$\begin{array}{ll}5 \text { Otimização da Resposta } & 67\end{array}$ 
5.1. Caso $1 \quad 67$

5.2. Caso 2

6 Síntese e Conclusões 72

6.1. Oportunidade de Melhoria e Recomendações para Futuras Pesquisas

Referências Bibliográficas 


\section{Lista de figuras}

Figura 1 - Região possível de misturas com 2 e 3 componentes (a e b respectivamente)

Figura 2 - Sistema de coordenadas trilinear

Figura 3 - Restrições a) inferiores e b) superiores nas proporções dos componentes

Figura 4 - Experimento da espuma do xampu 26

Figura 5 - Mistura de 3 componentes com 2 variáveis de processo $\quad 30$

Figura 6 - Experimento do adesivo aeroespacial 34

Figura $7-A I C_{c}$ mínimo em função do número de parâmetros 41

Figura 8 - Gráfico de probabilidade normal dos resíduos studentized $\quad 45$

Figura 9 - Gráfico de resíduos studentized versus run number $\quad 45$

Figura 10 - Gráfico de resíduos studentized versus valor ajustado $\quad 46$

Figura 11 - Gráfico para verificação de não constância da variância 46

Figura 12 - Gráfico de contorno da previsão da resposta para os modelos (2.9) e (2.10) 47

Figura 13 - Gráfico de contorno do desvio-padrão da média para os modelos (2.9) e (2.10)

Figura 14 - Gráfico de contorno da previsão da resposta para o Modelo Final (4.15)

Figura 15 - Gráfico de contorno do desvio-padrão da média para o Modelo Final (4.15)

Figura $16-A / C_{c}$ mínimo em função do número de parâmetros $\quad 50$

Figura $17-A / C_{c}$ mínimo em função do número de parâmetros 57

Figura 18 - Gráfico de probabilidade normal dos resíduos studentized 62

Figura 19 - Gráfico de resíduos studentized versus run number 62

Figura 20 - Gráfico de resíduos studentized versus valor ajustado $\quad 63$

Figura 21 - Gráfico para verificação de não constância da variância 63

Figura 22 - Gráficos de contorno da previsão da resposta para o modelo (3.8) 
Figura 23 - Gráficos de contorno do desvio-padrão da média para o modelo (3.8) 64

Figura 24 - Gráficos de contorno da previsão da resposta para o

Modelo Final (4.21) 65

Figura 25 - Gráficos de contorno do desvio-padrão da média para o Modelo Final (4.21) 66 


\section{Lista de tabelas}

Tabela 1 - Experimento da altura da espuma do xampu em

L-pseudocomponentes

Tabela 2 - Experimento da força do adesivo aeroespacial em

L-pseudocomponentes

Tabela 3 - $p$ versus $\min \left[\operatorname{AIC}_{c}(p)\right]$

Tabela 4 - Modelos indiferentes (M1 a M4) 42

Tabela 5 - Teste do Modelo (4.13) 42

Tabela 6 - Termos Equivalentes 43

Tabela 7 - Modelos indiferentes (M5 e M6) 43

Tabela 8 - Teste do Modelo Final (4.15) 44

Tabela 9 - Número de modelos em função do número de parâmetros 49

Tabela $10-p$ versus $\min \left[\operatorname{AIC}_{c}(p)\right] \quad 50$

Tabela 11 - Modelos indiferentes (M7 a M14) 52

Tabela 12 - Modelos indiferentes (M15 a M22) 53

Tabela 13 - Modelos indiferentes (M23 a M29) 54

Tabela 14 - Teste do Modelo (4.19) 55

Tabela 15 - Termos Equivalentes $\quad 56$

Tabela $16-p$ versus $\min \left[\operatorname{AIC}_{c}(p)\right] \quad 56$

Tabela 17 - Modelos indiferentes (M30 a M37) 58

Tabela 18 - Modelos indiferentes (M38 a M45) 59

Tabela 19 - Modelos indiferentes (M46 e M52) 60

Tabela 20 - Teste do Modelo Final (4.21) 61

Tabela 21 - Comparativo dos Modelos de Mistura 68

Tabela 22 - Valores dos componentes de mistura em

L-pseudocomponentes e actual componentes 68

Tabela 23 - Comparativo dos Modelos de Mistura-Processo 69 
Tabela 24 - Valores dos componentes de mistura em

L-pseudocomponentes e componentes reais e níveis das variáveis de processo

Tabela 25 - Comparativo dos Modelos de Mistura-Processo (Condição sugerida pelo experimentador)

Tabela 26 - Valores dos componentes de mistura em componentes reais e níveis das variáveis de processo (Condição sugerida pelo experimentador) 
"Pensar é o trabalho mais difícil que existe. Talvez por isso tão poucos se dediquem a ele."

Henry Ford 